\title{
FURTHER OPTICAL OBSERVATIONS OF HZ HERCULIS
}

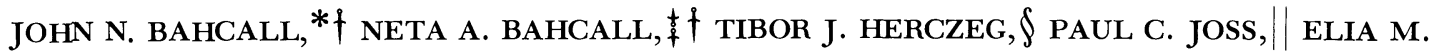 \\ LEIBOWITZ,,$\uparrow$ ALEX SEgALOVITZ, \# SHMUEL STOLERO,$\uparrow$ M. VERON,,$\uparrow$ PETER A. WEHINGER,,$\uparrow$ \\ DONNA WEISTROP, $\nmid$ AND SUSAN WYCKOFF $\nmid$ \\ Received 1974 October 10
}

\begin{abstract}
Optical observations of HZ Her made in the spring and summer of 1973 are presented. Some of the observations are practically coincident with estimated turn-on times for Her X-1 and others are simultaneous with $\mathrm{X}$-ray observations made using the Copernicus satellite. Eight magnitudes were also measured from old plates for the epoch 1953-60; these measurements confirm the long-term variations for that epoch reported by Jones, Forman, and Liller (1973). An interesting theoretical explanation by Fabian, Pringle, and Rees (1973) for the persistence of large optical variations throughout the 35-day $\mathrm{X}$-ray period is ruled out by simultaneous UHURU (X-ray) and Wise Observatory (optical) observations.
\end{abstract}

Key words: X-ray source - binary star - photometry

\section{Introduction}

The binary system consisting of $\mathrm{HZ}$ Herculis and Her X-1 has been the subject of many theoretical and observational investigations following the initial discovery that it contained a periodically pulsing $\mathrm{X}$-ray source (Schreier et al. 1972), the suggestion by Liller (1972) that $\mathrm{HZ} \mathrm{Her}$ was an interesting candidate for the optical source, and the positive identification by Bahcall and Bahcall (1972a). Some of the moredetailed optical observations are described by Bahcall and Bahcall (1972b), Davidsen et al. (1972), Forman, Jones, and Liller (1972), Bopp, Grupsmith, and Vanden Bout (1972), Crampton and Hutchings (1972), Groth and Nelson (1972), Lyutiy, Sunyaev, and Cherepashchuk, (1973), Petro and Hiltner (1973), Boynton et al. (1973), Bopp et al. (1973), Cocke et al. (1973), Chevalier and Ilovaisky (1973), Crampton (1974), Groth (1974).

We present in section II our new observational results (see Fig. 1) which include $76 \mathrm{~B}$ magnitudes measured during the spring and summer of 1973 at the Wise Observatory, as well as magnitudes measured on eight nights in the years 1953-60 with the University of Oklahoma astrographic telescope. Some theoretical in-

*Institute for Advanced Study, Princeton; †Tel Aviv University, Ramat Aviv, Israel; + Princeton University; §The University of Oklahoma; ||Massachusetts Institute of Technology; \#Sterrewacht te Leiden, Leiden, The Netherlands. ferences are presented in section III. Of special interest are our observations that are practically coincident with the estimated turn-on times for Her X-1 in 1973 May and August. We also report observations in 1973 August that were simultaneous with X-ray observations made using the Copernicus satellite (Triton 1973; Fabian and Hoffman 1973).

Our observations (see Table I) made during 1953 to 1960 confirm the long-term variations of that epoch reported by Jones, Forman, and Liller (1973). In agreement with their results we find that HZ Her was in a low-intensity state for the nights in 1953, 1954, and 1956 that are covered by our measurements. In addition, HZ Her was bright on the one night in 1960 covered by the observations.

\section{Observations}

\section{A. 1973 Observing Season}

In Figure 1 we show the observed $B$ magnitudes measured for 76 separate times during the period from 1973 March 1 to 1973 August 26. The results were obtained using the 1-meter Ritchey-Chrétien reflector telescope of the Wise Observatory with Kodak 103a-O plates exposed for 15 minutes behind a Wratten-47 filter. The magnitude system and the reduction procedure are the same as described by Bahcall and Bahcall (1972b); the typical rms error for an individual magnitude is $0^{\mathrm{m}} 1$.

The turn-on times (UT dates) for the 35-day 

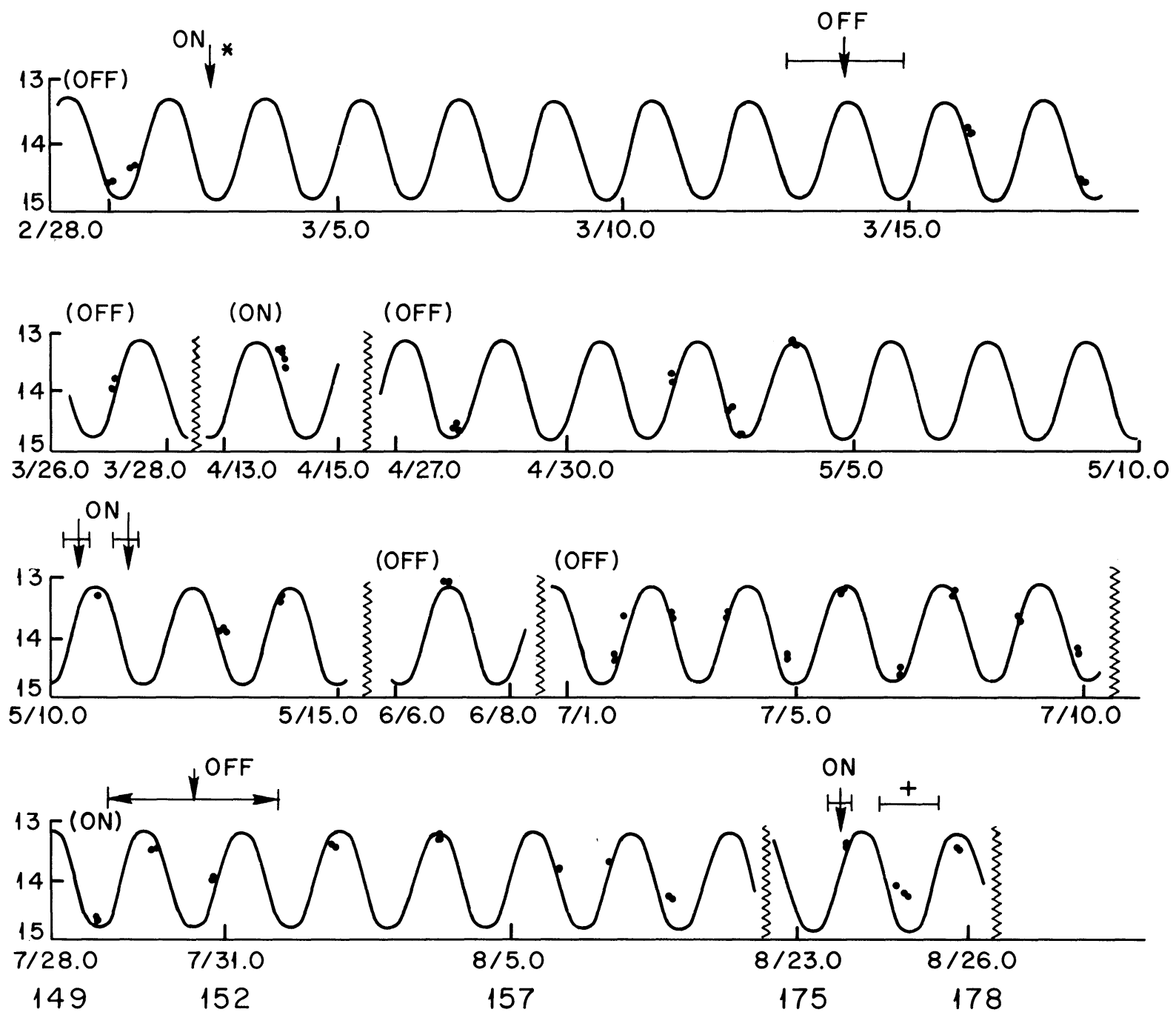

FIG. $1-B$ magnitudes for 1973. The measured magnitudes are shown as black dots. During the period marked by † Her X-1 was observed to be "on" by the Copernicus satellite (Fabian and Hoffman 1973). The time marked by a * indicates that Her X-1 was observed to turn-on by the UHURU satellite (Tananbaum et al. 1973).

cycle of the X-ray source were calculated (cf. Groth 1974) using the observed turn-on times, 1973 March $1.72 \pm 0.04$ (see Tananbaum et al. 1973) and 1973 August $24.1 \pm 1.04$ (Tananbaum et al. 1973; Fabian and Hoffman 1973), plus the assumption (see Giacconi et al. 1973) that the turn-on times are quantized in units of approximately half the orbital period (i.e., the time between successive turn-ons is (1.70 [ 20 $+n / 2] \pm 0.2$ ) days, $n=0,1$, or 2). These considerations uniquely fix the August turn-on time at August $23.8 \pm 0.2 \mathrm{UT}$. The X-ray source gradually becomes unobservable (turn-off) $11 \pm 1$ days after each turn-on. Thus, the total uncertainty in the March turn-off is \pm 1 day and the total uncertainty in the July/August turn-off time is \pm 1.4 days (see Fig. 1). If the behavior of the 35-day cycle during the spring and summer of 1973 was the same as that suggested by the observations of Giacconi et al. (1973), then there are no additional uncertainties in the turn-on and turn-off times shown on Figure 1. (Of course, we cannot rule out the possibility of different behavior, e.g., the occurrence of values of $n$ other than 0,1 , and 2.)

We note (see Fig. 1) that there are no very large departures from the previously determined (Bahcall and Bahcall 1972b) average 
light curve. $\mathrm{HZ} \mathrm{Her}$ was in its variable state from 1973 March through August.

B. Some Observations in 1953-60

We give, in Table I, 18 individual magnitudes observed for $\mathrm{HZ}$ Her on eight separate nights during the years 1953 to 1960 . The plates used (also Kodak 103a-O) were taken by B. Whitney with the $8.3-\mathrm{cm}$ astrograph of the University of

TABLE I

Some Magnitudes of HZ Herculis 1950-1960

\begin{tabular}{|c|c|c|c|c|c|}
\hline Plate No. & Year & $\begin{array}{l}\text { Julian Date } \\
2,400,000+\end{array}$ & Phase & $\mathrm{m}_{\mathrm{B}}$ & Remarks \\
\hline $\mathrm{R} 7558$ & 1953 & (24) 34595.69 & 0.29 & 14.6 & \\
\hline R 7638 & 1953 & 34632.58 & 0.98 & $(14.6)$ & estimated \\
\hline R 8022 & 1954 & 34829.81 & 0.99 & 14.54 & \\
\hline R 8023 & 1954 & 34829.83 & 0.00 & 14.54 & \\
\hline R 8207 & 1954 & 34925.70 & 0.39 & 14.95 & \\
\hline R 8208 & 1954 & 34925.72 & 0.40 & 14.43 & \\
\hline R 8934 & 1955 & 35392.59 & 0.00 & $(14.7)$ & estimated \\
\hline R 8935 & 1955 & 35392.60 & 0.01 & 14.5 & \\
\hline R 8936 & 1955 & 35392.61 & 0.02 & 14.7 & \\
\hline R 8937 & 1955 & 35392.63 & 0.025 & $(14.7)$ & estimated \\
\hline $\mathrm{R} 9309$ & 1956 & 35605.755 & 0.38 & 14.7 & \\
\hline R 9310 & 1956 & 35605.77 & 0.39 & 14.8 & \\
\hline R 9311 & 1956 & 35605.78 & 0.40 & 14.6 & \\
\hline R 9438 & 1956 & 35698.73 & 0.07 & 14.4 & \\
\hline $\mathrm{R} 9439$ & 1956 & 35698.74 & 0.076 & $(14.6)$ & faint image \\
\hline R 9440 & 1956 & 35698.75 & 0.082 & 14.5 & \\
\hline R 11690 & 1960 & 37115.65 & 0.46 & 13.37 & \\
\hline R 11691 & 1960 & 37115.66 & 0.47 & $(13.0)$ & extrapolated \\
\hline
\end{tabular}


Oklahoma and were reduced by one of us (T.J.H.) according to the magnitude system of Bahcall and Bahcall (1972b).

\section{Position}

The position of $\mathrm{HZ}$ Her has been measured with the Mann x-y comparator of Tel Aviv University on an epoch 1972 plate (no. MR-692) taken at Wise Observatory. Using a series of reference stars and the standard reduction procedure of Véron and Véron (1973), we find the position of HZ Her to be

$$
\alpha(1950)=16^{\mathrm{h}} 56^{\mathrm{m}} 01 \text { s } 67, \delta(1950)=+35^{\circ} 25^{\prime} 05^{\prime \prime} 1
$$

with a probable error of 0.3 in either coordinate.

\section{Discussion}

\section{A. Phase Lag and Asymmetry}

The $1 \mathrm{~d} 7$ periodicities of the X-ray and optical light curves are equal to high accuracy (see especially Jones et al. 1973); they result from the orbital period, $P$, of the binary system. The numerical value of $P$ is known very accurately from the X-ray observations (Tananbaum et al. 1972; Giacconi et al. 1973). It has also been shown that the optical light curve is fitted remarkably well by a simple law of the form $L_{B}=L_{0}(1-A \cos 2 \pi \phi)$, where $L_{B}$ is the intensity in the $B$-band, $\phi$ is the $\mathrm{X}$-ray orbital phase, and $L_{0}$ and $A$ are constants (Bahcall and Bahcall 1972b; Bahcall, Joss, and Avni 1974). We have combined the 1973 data with the Wise Observatory data of 1972 (Bahcall and Bahcall $(1972 b)$ plus a few additional data of 1972 September) to set an improved limit on a possible phase lag between optical and X-ray light curves (cf. Bahcall et al. 1974); this limit constitutes a valuable constraint on theoretical models.

We define the phase lag, $\delta$, by the light curve $L_{B}=L_{0}[1-A \cos 2 \pi(\phi-\delta / P)]$, which is symmetric in shape [i.e., $L_{B}(\phi-\delta / P)=L_{B}(-[\phi-$ $\delta / P])]$. Fitting our total of 159 data points to a light curve of this form, we find that $A=0.57$ \pm 0.02 and $\delta=0.003 \pm 0.010$ day (approximate single parameter $95 \%$ confidence limits). Thus, $|\delta| \lesssim 0.01$ day $=15$ minutes.

If we allow for a possible asymmetry in the light curve [i.e., $\quad L_{B}(\phi-\delta / P) \neq L_{B}(-[\phi-$ $\delta / P])]$, the limit on the phase lag becomes less precise. For example, when we fit our data to light curves of the form.

$$
\begin{aligned}
L_{B}= & L_{0}\left[1-A_{1} \cos 2 \pi\left(\phi-\delta_{1} / P\right)\right. \\
& \left.+B_{1} \sin 4 \pi \phi\right]
\end{aligned}
$$

or

$$
\begin{aligned}
L_{B}= & L_{0}\left[1-A_{2} \cos 2 \pi\left(\phi-\delta_{2} / P\right)\right. \\
& \left.+B_{2} \sin 4 \pi\left(\phi-\delta_{2} / P\right)\right],
\end{aligned}
$$

we obtain, respectively,

$$
\begin{aligned}
A_{1} & =0.57 \pm 0.02, B_{1}=0.023 \pm 0.029 \\
\delta_{1} & =0.011 \pm 0.014 \text { day }
\end{aligned}
$$

and

$$
\begin{aligned}
A_{2} & =0.57 \pm 0.02, B_{2}=0.016 \pm 0.013 \\
\delta_{2} & =0.035 \pm 0.028 \text { day }
\end{aligned}
$$

(For the present data, additional terms in the Fourier-series expansion of $L_{B}$ are generally consistent with zero.) Thus, there is some marginal evidence for an asymmetry and for the suggestion that the optical minimum (or maximum) follows the $\mathrm{X}$-ray minimum (or maximum). However, both these marginal suggestions are dependent upon estimates of errors made with an average light curve that has no 35-day dependence included; more precise photometric data (now available from several groups) should be used to test for small asymmetries and phase lags.

\section{B. "On" and "Off" States}

Finally we note that the original observations at Wise Observatory can rule out an interesting theoretical suggestion recently made by Fabian, Pringle, and Rees (1973). These authors propose that the apparent absence of a very large 35-day effect in the optical light curve may be explained by assuming that the $\mathrm{X}$-ray source was really "on" at UHURU energies whenever the optical observations were being made. They note that the published UHURU observations made after the optical identification of $\mathrm{HZ}$ Her were always performed during "on" periods. However, UHURU observations were also made prior to turn-on times in order to determine the onset times accurately. Schreier (1974) has kindly informed us that UHURU detected no measurable X-ray emission from Her X-1 on 1972 August 4.97, $5.25,5.35,5.95$, and 6.03 (UT dates) prior to the 
X-ray turn-on at August 6.99. Bahcall and Bahcall (1972b) (see their Fig. 1) report seven observations each night of $\mathrm{HZ}$ Her from 1972 August 4.78 to 4.92 and on 1972 August 5.76 to 5.94. On both nights, the light curve of $\mathrm{HZ} \mathrm{Her}$ was normal, contrary to the suggestion of Fabian et al. (1973).

The Wise Observatory derives partial support from the Smithsonian Research Foundation through Grant no. SFC-0-3005.

The work of J. N. and N. A. Bahcall is partially supported by NSF contract 32394 with Princeton University.

The work of J. N. Bahcall is partially supported by NSF Grant no. GP-40768X.

\section{REFERENCES}

Bahcall, J. N. and Bahcall, N. A. 1972a, I.A.U. Circ. Nos. 2427 and 2428.

- 1972b, Ap. J. (Letters) 178, L1.

Bahcall, J. N., Joss, P. C., and Avni, Y. 1974, Ap. J. 191, 211.

Bopp, B. W., Grupsmith, G., and Vanden Bout, P. 1972, Ap. J. (Letters) 178, L5.

Bopp, B. W., Grupsmith, G., McMillan, R. S., Vanden Bout, P. A., and Wooten, H. A. 1973, Ap. J. (Letters) 186, L123.

Boynton, P. E., Canterna, R., Crosa, L., Deeter, J., and Gerend, D. 1973, Ap. J. 186, 617.

Chevalier, C., and Ilovaisky, S. A. 1973, Nature Phys. Sci. $245,87$.

Cocke, W. J., Hintzen, P., Scott, J. S., and Worden,
S. P. 1973, Nature Phys. Sci. 244, 137.

Crampton, D. 1974, Ap. J. 187, 345.

Crampton, D., and Hutchings, J. B. 1972, Ap. J. (Letters) 177, L103.

Davidsen, A., Henry, J. P., Middleditch, J., and Smith, H. E. 1972, Ap. J. (Letters) 177, L97.

Fabian, A. C., and Hoffman, J. A. 1973, I.A.U. Circ. No. 2581.

Fabian, A. C., Pringle, J. E., and Rees, M. J. 1973, Nature 244, 212.

Forman, W., Jones, C. A., and Liller, W. 1972, Ap. J. (Letters) 177, L103.

Giacconi, R., Gursky, H., Kellogg, E., Levinson, R., Schreier, E., and Tananbaum, H. 1973, Ap. J. 184, 227.

Groth, E. J. 1974, Ap. J. 192, 517.

Groth, E. J., and Nelson, M. R. 1972, Ap. J. (Letters) 178, L111.

Jones, C. A., Forman, W., and Liller, W. 1973, Ap. J. (Letters) 182, L109.

Liller, W. 1972, I.A.U. Circ. No. 2415.

Lyutiy, V. M., Sunyaev, R. A., and Cherepashchuk, A. M. 1973, Astr. Zu. 50, 3.

Petro, L., and Hiltner, W. A. 1973, Ap. J. (Letters) 181, L39.

Schreier, E. 1974 (private communication).

Schreier, E., Levinson, R., Gursky, H., Kellogg, E., Tananbaum, H., and Giacconi, R. 1972, Ap. J. (Letters) 172, L79.

Tananbaum, H., Gursky, H., Kellogg, E. M., Levinson, R., Schreier, E., and Giacconi, R. 1972, Ap. J. (Letters) 174, L143.

Tananbaum, H, Schreier, E., Levinson, R., Giacconi, R., Gursky, H., Kellogg, E., and Matilsky, R. 1973, I.A.U. Circ. No. 2578.

Triton, K. P. 1973 (private communication).

Veron, M., and Veron, P. 1973 Astr. and Ap. 28, 319. 
